\title{
Erasure-Coding Based Routing for Opportunistic Networks
}

\author{
Yong Wang, Sushant Jain ${ }^{\dagger}$, Margaret Martonosi, Kevin Fall ${ }^{\star}$ \\ Princeton University, †University of Washington, ‡Intel Research Berkeley
}

\begin{abstract}
Routing in Delay Tolerant Networks (DTN) with unpredictable node mobility is a challenging problem because disconnections are prevalent and lack of knowledge about network dynamics hinders good decision making. Current approaches are primarily based on redundant transmissions. They have either high overhead due to excessive transmissions or long delays due to the possibility of making wrong choices when forwarding a few redundant copies. In this paper, we propose a novel forwarding algorithm based on the idea of erasure codes. Erasure coding allows use of a large number of relays while maintaining a constant overhead, which results in fewer cases of long delays.

We use simulation to compare the routing performance of using erasure codes in DTN with four other categories of forwarding algorithms proposed in the literature. Our simulations are based on a real-world mobility trace collected in a large outdoor wild-life environment. The results show that the erasure-coding based algorithm provides the best worst-case delay performance with a fixed amount of overhead. We also present a simple analytical model to capture the delay characteristics of erasure-coding based forwarding, which provides insights on the potential of our approach.
\end{abstract}

\section{Categories and Subject Descriptors}

C.2.2 [Network Protocols]: Routing protocols

\section{General Terms}

Algorithms, Performance, Theory

\section{Keywords}

Routing, Delay Tolerant Network, Erasure Coding

\section{INTRODUCTION}

Opportunistic networks are an important class of DTNs in which contacts (time-window when data can be exchanged) appear $o p$ portunistically without any prior information. Examples of such networks are sparse mobile ad hoc networks, such as ZebraNet [8],

Permission to make digital or hard copies of all or part of this work for personal or classroom use is granted without fee provided that copies are not made or distributed for profit or commercial advantage and that copies bear this notice and the full citation on the first page. To copy otherwise, to republish, to post on servers or to redistribute to lists, requires prior specific permission and/or a fee.

SIGCOMM'05 Workshops, August 22-26, 2005, Philadelphia, PA, USA.

Copyright 2005 ACM 1-59593-026-4/05/0008 ...\$5.00. where no contemporaneous end-to-end path may exist due to radio range limitations. Routing becomes challenging in such networks because contact dynamics are not known in advance and no single path can be relied upon. Most current approaches are based on some kind of data replication over multiple paths $[14,8]$. In this paper, we propose an alternate method of improving delay performance. The basic idea is to erasure code a message and distribute the generated code-blocks over a large number of relays. Compared to sending a full copy of the message over a relay, only a fraction of code-blocks are sent over each relay. This fraction allows us to control the routing overhead in terms of bytes transmitted. For scenarios like ZebraNet, where nodes are energy constrained, limiting such overhead is an important design goal.

The basic idea of using erasure coding is simple and has been explored in many applications [11]. However, it is not clear if and when it will perform better than simpler alternatives based on pure replications in DTNs. In this paper, we study the performance of an erasure coding approach and other existing alternatives on a diverse mobility scenarios with different node densities and moving patterns. We use both synthetic and real-world DTN mobility traces as input to our simulations. We discover that the erasure coding approach can provide good delay guarantees by using a fixed overhead. Fundamentally, the benefits of erasure coding arise in eliminating cases when long delays arise due to bad choice of forwarding relays. Erasure coding allows the transmission to be spread over multiple relays while using a fixed amount of overhead. This results in a protocol much more robust to failures of a few relays or some bad choices. We find that the erasure-coding based algorithm is the least sensitive to different parameters in terms of message latency and message delivery rate. Also, we derive an expression for the delay distribution under a simple network model to argue when and why the erasure coding approach outperforms other simpler alternatives. In one extreme case, we show that the average delay of a simple replication strategy will be infinite, whereas, by using erasure coding the average delay can be reduced to a small constant.

Erasure coding can also help combat packet loss due to bad channel quality or packet drops due to congestion. A full investigation of the benefits of this aspect is outside the scope of this paper. Here, our focus is on a less-conventional use of erasure coding: to achieve better delay performance using a fixed amount of replication.

\section{BACKGROUND}

In an opportunistic network, reliable data delivery is often achieved using replication to send identical copies of a message over multiple paths to mitigate the effects of disconnections. Typical algorithms differ based on their decisions as to who forwards the data, at what time is the data forwarded, and to whom is the data sent. In 
the following discussions, we define a contact as an opportunity to communicate between two nodes and a relay denotes a forwarding node.

- Flooding (flood): each node forwards any non duplicated messages (including messages received on behalf of other nodes) to any other node that it encounters. flood delivers messages with the minimum delay if there are no resource constraints, such as link bandwidth or node storage.

- Direct contact (direct): the source holds the data until it comes in contact with the destination. direct uses minimal resources since each message is transmitted at most once. However, it may incur long delays.

- Simple replication $(\operatorname{srep}(r))$ : this is a simple replication strategy in which identical copies of the message are sent over the first $r$ contacts. Here, $r$ is the replication factor. Only the source of the message sends multiple copies. The relay nodes are allowed to send only to the destination; they cannot forward it to another relay. This leads to small overhead as the message flooding is controlled to take place only near the source. This class of forwarding algorithms is also known as the two-hop relay algorithm $[3,2]$. There is a natural trade-off between overhead $(r)$ and data delivery latency. A higher $r$ leads to more storage/transmissions but has lower delays.

- History-based (history $(r)$ ): here history is used as an indicator of the probability of delivery. Each node keeps track of the probability that a given node will deliver its messages. $r$ highest ranked relays (based on delivery probability) are selected as forwarding nodes. ZebraNet uses the frequency at which a node encounters destination as an indicator of the delivery probability. We use the same implementation as [8] in our simulations.

A summary of these forwarding algorithms is listed in Table 1.

\begin{tabular}{|c|ccc|}
\hline Algorithm & Who & When & To whom \\
\hline flood & all nodes & new contact & all new \\
direct & source only & destination & destination only \\
srep $(r)$ & source only & new contact & $r$ first contacts \\
history $(r)$ & all nodes & new contact & $r$ highest ranked \\
\hline
\end{tabular}

Table 1: Summary of various forwarding algorithms.

\section{THE ERASURE-CODING BASED FOR- WARDING ALGORITHM}

As discussed in the previous section, most current approaches for routing in opportunistic networks are based on sending multiple identical copies over different paths. There is a fundamental trade-off between overhead and delay. On one extreme, flooding achieves the best possible delay but results in very high overhead. The other extreme is protocols like direct which have low overhead because they send only few copies or none at all. Lack of knowledge about the topology dynamics prevents distinguishing good paths from bad ones. Therefore, these protocols may result in long delays if bad paths are selected. In this section, we describe a forwarding algorithm based on the idea of erasure coding. Our algorithm achieves better worst-case delay performance than existing approaches with a fixed overhead.

\subsection{Erasure coding background}

Erasure codes operate by converting a message into a larger set of code blocks such that any sufficiently large subset of the generated code blocks can be used to reconstruct the original message. More precisely, an erasure encoding takes as input a message of size $M$ and a replication factor $r$. The algorithm produces $M * r / b$ equal sized code blocks of size $b$, such that any $(1+\epsilon) \cdot M / b$ erasure coded blocks can be used to reconstruct the message. Here, $\epsilon$ is a small constant and varies depending on the exact algorithm used, such as Reed-Solomon codes or Tornado codes. The selection of algorithms involve trade-offs between coding/decoding efficiency and the minimum number of code blocks to reconstruct a message. For example, Tornado codes have efficient encoding and decoding steps based on simple operations such as XOR, at the cost of slightly higher $\epsilon$. A thorough discussion of the various trade-offs is presented in [11]. The choice of exact erasure coding algorithm is not important in our forwarding algorithm. The key aspect is that when using erasure coding with a replication factor of $r$, only $1 / r$ of the code blocks are required to decode the message. Therefore, we ignore constant $\epsilon$ for simplicity. Constant $b$ is the block-size and is implementation dependent.

\subsection{Erasure coding based forwarding (ec)}

Our erasure-coding based forwarding algorithm can be understood as an enhancement to the simple replication algorithm (srep) described in Section 2.

In srep with a replication factor $r$, the source sends $r$ identical copies over $r$ contacts and relays are only allowed to send directly to the destination. In the erasure-coding based algorithm, we first encode the message at the source and generate a large number of code blocks. The generated code blocks are then equally split among the first $k r$ relays, for some constant $k$. In comparison with srep, this approach uses a factor of $k$ more relays and each relay carries a factor of $1 / k$ less data. However, the number of bytes generated are $r M$, the same as the number of bytes generated by srep (r).

Now by definition of erasure coding (with rate $r$, message size $M)$, the message can be decoded at the destination if $1 / r$ of the generated code blocks are received. Since code blocks are divided equally among $k r$ relays, the message can be decoded as soon as any $k$ relays deliver their data if we assume that no code blocks are lost during transmissions to and from a relay.

When $k=1$, the erasure coding approach has the same effect as the simple replication approach, which is, to use the first $r$ relays and to each carry a copy of the original message.

\subsection{Benefits of erasure coding in forwarding}

In simple replication, $r$ relays are used to improve the delay performance. The erasure-coding based approach, instead, utilizes $k r$ relays for the same amount of overhead. Therefore, one can expect that the chances of at least some relays having low delays are higher, compared to using only $r$ relays. At the same time, erasure coding requires at least $k$ relays to succeed (instead of 1 in srep) before the data can be reconstructed. Therefore, if the number of such low-delay relays are larger than $k$, the erasure-coding based approach will successfully deliver the message with a lower delay than simple replication. Thus, the fundamental question is whether to use $r$ relays and wait for one to succeed or use $r * k$ relays and wait for $k$ relays to succeed. We answer this question using a simple analytical model in Section 5. The main observation is that if $k$ is large, the delay distribution converges to a constant. Therefore, with the erasure-coding based approach, one can be almost assured of a constant delay. 


\section{EVALUATION}

In this section, we use simulation to compare forwarding algorithms discussed in Section 2 and the erasure-coding based approach presented in Section 3.

\subsection{Methodology}

We use dtnsim, the discrete event simulator for DTN environments from [6]. We implemented the following routing algorithms in dtnsim: flooding (flood), direct contact routing (direct), history-based routing (hi story), simple replication routing (srep) and erasure-coding based routing (ec). For srep and ec, we represent different replication factors and number of relays used to split, using srep-rep $r$ and ec-rep $r$-p $n$. Here, $r$ is the replication factor and $n$ are the number of relays among which code blocks are divided.

We simulate using a real-world mobility trace collected as part of a wildlife tracking experiment in Kenya. The mobile network was deployed by the ZebraNet group in January, 2004 [15]. Tracking collars are placed on the necks of selected zebras. Each collar uses GPS to record its position data every 8 minutes, and periodically sends back position log data to a mobile base station (e.g., a vehicle). Due to extreme weather and waterproofing issues, as well as antenna problems, only one tracking collar returned a complete set of uninterrupted movement data for the whole 32-hour duration. Due to such limitations ${ }^{1}$, we create a semi-synthetic mobility model as follows: we synthesize node speed and turn angle distributions from the observed data and create other node movements following the same distribution. We scale the grid size to $6 \mathrm{~km} \times 6 \mathrm{~km}$ with a radio range of $1 \mathrm{~km}$. Initially, the nodes are randomly distributed in the grid. The base station moves along a rectangular path near the grid boundary. All messages are of size $1 \mathrm{M}$. Each node generates 12 messages every day. The total duration of simulation is 16 days. Another mobility model based on heavytailed inter-contact times is discussed in Section 4.4.

We compare the routing performance of different forwarding algorithms using the following three metrics:

- Data success rate: the ratio of the number of messages that are delivered to the destination within a time $T$ (deadline). If $T$ is unspecified, it is considered to be the whole duration of the simulation, i.e 16 days.

- Data latency: the duration between message generation and message reception (at its destination). In a DTN, latency may not be the most critical issue. However, it is always desirable to have fast data delivery whenever possible. The latency distribution metric measures how efficiently a protocol uses the available contact opportunities.

- Routing overhead: the ratio of the number of bytes transmitted to the number of bytes generated during the simulation time. This metric measures the extra data transmitted for each message generated, while a metric based solely on the number of message transmissions will overlook the fact that ec has smaller message sizes. The radio transmission energy is proportional to the total number of byes transmitted. Therefore, this metric reflects the energy efficiency of the forwarding algorithm.

\footnotetext{
${ }^{1}$ At the moment, we are working on collecting more node traces during our second field trip in June, 2005. We will work on adjusting the model once we have those node traces available.
}

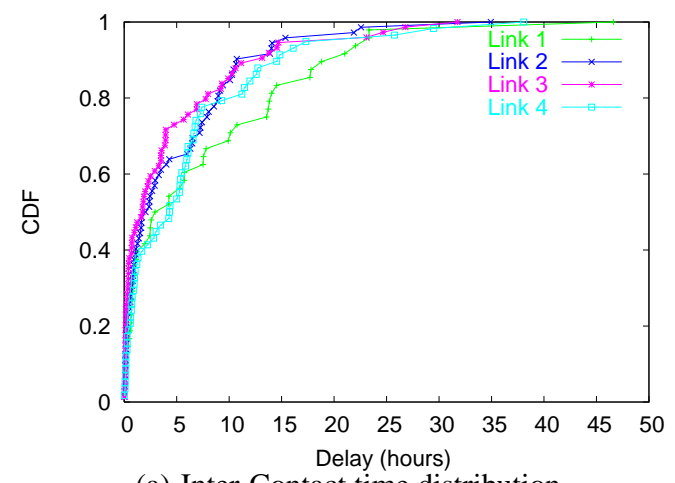

(a) Inter-Contact time distribution

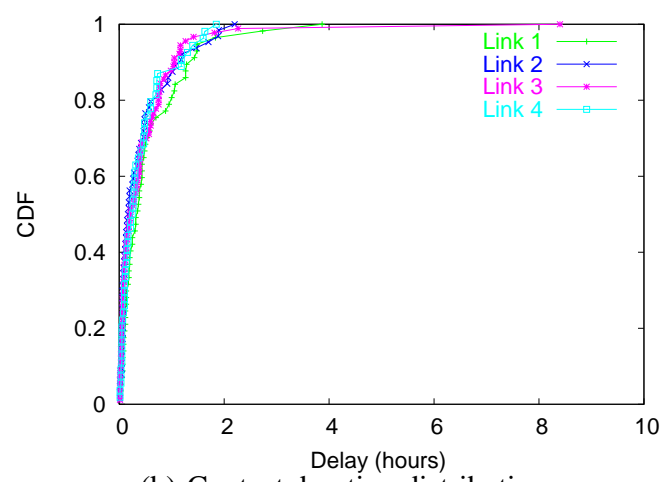

(b) Contact duration distribution

Figure 1: Cumulative distribution plots for inter-contact times and contact durations for the ZebraNet trace. The figure plots these two metrics for four randomly selected links. Other links show similar characteristics. The contact duration distribution uses a different $x$-axis range to separate different curves. Observe that inter-contact time patterns show significant variation and can be very long in some cases.

\subsection{Zebra trace analysis}

To begin our analysis, we first characterize the contact opportunities in the ZebraNet trace, with a focus on inter-contact time and contact durations. These two metrics are important in understanding the behavior of different forwarding algorithms on the ZebraNet trace. Simply put, inter-contact time is the time interval for which a link is down (no communications are possible during this time) and contact duration is the interval for which a link is up.

Figure 1 plots the distribution of these two metrics for four randomly selected pairs of nodes (links) in the ZebraNet trace. Since almost all the links in the trace show similar characteristics, we just use these four random links as examples.

As shown in Figure 1(a), the inter-contact time distribution has few cases when a link is broken for a very long time. This observation is important because such inter-contact time patterns can lead to extremely long delays when using a naive forwarding algorithm. As expected in such a sparse network, link up-times are relatively short (as compared to the link down times) and therefore, it is important to efficiently utilize the available communication opportunity.

\subsection{Impact of node density}

\subsubsection{Data latency distribution}

Figure 2(a) and 2(b) show the data latency distribution for the 


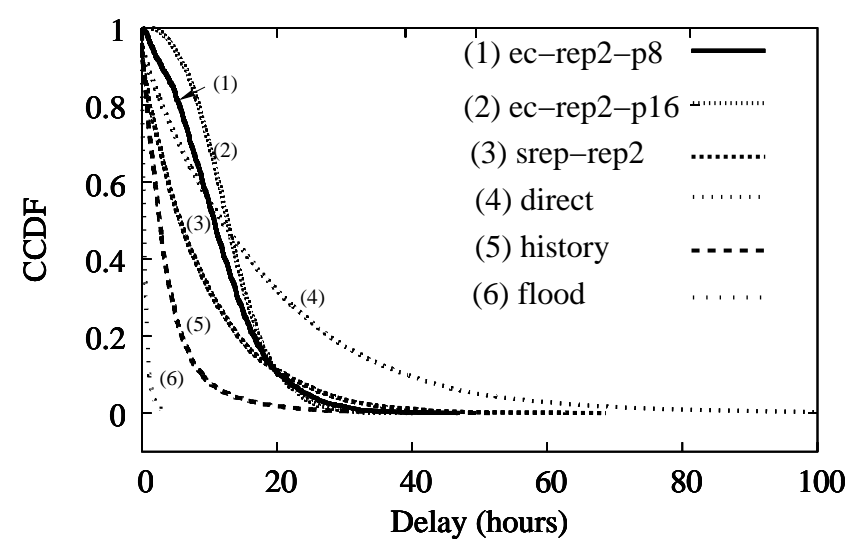

(a) 34 nodes

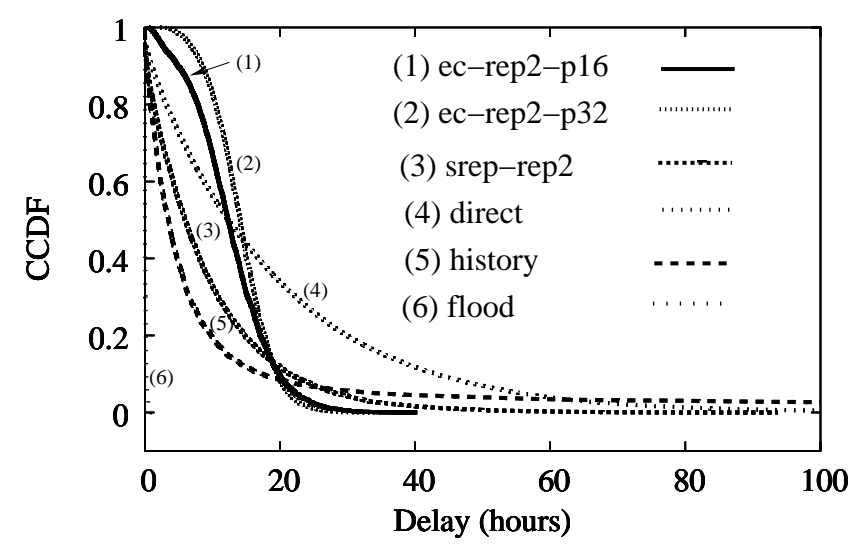

(b) 66 nodes

Figure 2: Latency distribution for different forwarding algorithms. Traffic injection rate is 12 messages per day. The distribution is shown in Complementary CDF (CCDF) curve. A numeric presentation of this figure is in Table 2 which lists the exact $50^{t h}, 90^{t h}$ and $99^{t h}$ percentiles delay. The erasurecoding based approach has significantly smaller tail than other approaches (except flood). flood has the lowest latencies but has high overhead as discussed later.

ZebraNet trace with 34 nodes and 66 nodes respectively. Discounting source and destination, the total number of relays are 32 and 64 respectively. The distribution is shown in Complementary CDF (CCDF) curve.

Table 2 shows various data latency percentiles for both 34-node and 66-node experiments to facilitate the comparison of worse-case delay performance among all the algorithms considered.

Generally, ec has a higher $50^{t h}$ percentile compared to other algorithms as shown in both Figure 2(a) and Figure 2(b) but a lower $99^{\text {th }}$ percentile. This is because it takes longer to find enough relays to distribute data replicas. However, once ec distributes enough code blocks by forwarding along multiple relays (the number of relays is larger than that used by srep), it takes a much shorter time to transfer the messages to the destination since any $n / r$ relays are required to be successful. Since $n$ is much larger than $r$, ec can fully utilize the diversity of multiple relays and is very robust to bad performance of individual relays. That is, in the presence of unpredicted failures or mobility of some of the relays, ec still has a good chance of sending the messages to the destination by routing code blocks through other functional relays.

\begin{tabular}{|c|ccc|ccc|}
\hline \multirow{2}{*}{ Algorithm } & \multicolumn{3}{|c|}{34 nodes } & \multicolumn{3}{c|}{ 66 nodes } \\
& $50 \%$ & $90 \%$ & $99 \%$ & $50 \%$ & $90 \%$ & $99 \%$ \\
\hline ec-rep2-p8 & 0.44 & 0.84 & 1.32 & - & - & - \\
ec-rep2-p16 & 0.53 & 0.85 & 1.21 & 0.51 & 0.83 & 1.17 \\
ec-rep2-p32 & - & - & - & 0.59 & 0.82 & 1.04 \\
srep-rep2 & 0.24 & 0.88 & 1.70 & 0.25 & 0.89 & 1.91 \\
direct & 0.49 & 1.63 & 3.27 & 0.51 & 1.79 & 3.54 \\
history & 0.18 & 0.87 & 9.50 & 0.14 & 0.72 & 10.83 \\
flood & 0.013 & 0.044 & 0.12 & 0.00012 & 0.0091 & 0.032 \\
\hline
\end{tabular}

Table 2: Latency (in days) for different algorithms for two different node densities. This is the same data as shown in Figure 2. We see that ec has significantly lower $99^{t h}$ percentile latency. This indicates that $e_{c}$ is effective in getting rid of very high latency cases.

Therefore, erasure-coding based routing is a promising candidate for opportunistic networks where (1) relay failures are prevalent and delays are unpredictable, and (2) minimizing the worst-case delay is important.

This observation is further supported by the data shown in Figure 2(b) where node density is higher. Given more contacts and relays, the CCDF curves of all forwarding algorithms become steeper. This is because there are more contacts overall. ec, as we have explained, still has the lowest $99^{t h}$ percentile and the sharpest data latency curve. Therefore, given enough relay opportunities, ec has the best performance in delivering most of the messages the fastest among all the algorithms considered.

Simple replication, direct contact, and history-based algorithms, on the other hand, have very long tails (messages with much longer delays). This is because they use a small number of relays. Therefore, they cannot guarantee when these relays will see the destination. Very likely, some packets may encounter very long delays by selecting some relays that fail to deliver the message promptly. In the long run however, with sufficient buffer space, all messages will eventually be delivered. The lower the replication factor $r$, the longer the tail will be. This is illustrated by comparing the CCDF of srep-rep2 and direct. Since srep-rep2 replicates its data to two other relays, the chance of losing contact opportunities is lower than that for direct. Hence, srep-rep2 has a shorter tail than direct.

The history approach, though having the lowest $50^{\text {th }}$ percentile delay, also has the longest tail among all the algorithms considered. The performance of history is dependent on the accuracy of its selection of highest ranked relays based on past statistics. If the decision is relatively accurate, it tends to find relays that will forward the data to the destinations very quickly. On the contrary, if the relays selected based on this heuristic do not reflect future forwarding probabilities, very long delays may be incurred. However, using certain timeout and retransmission schemes, these long-delay messages might be masked out which makes the history approach more attractive over the others in networks with predictable node movement. This is an interesting research direction to explore.

Finally, observe that the flood protocol in Figure 2(a) and Figure 2(b), has latency distribution curves which are almost vertical. This shows that flood has very low delays for all messages.

\subsubsection{Routing overhead}

Table 3 lists the routing overhead corresponding to each forwarding algorithm. Routing overhead is measured using the ratio of bytes transmitted to the bytes generated. Since both ec and srep transmit a fixed amount of data with respect to the data generated, 


\begin{tabular}{|c|cc|}
\hline \multirow{2}{*}{ Algorithm } & \multicolumn{2}{|c|}{ Overhead } \\
& (34 nodes) & (66 nodes) \\
\hline ec-rep2-p8 & 3.96 & - \\
ec-rep2-p16 & 3.96 & 3.98 \\
ec-rep2-p32 & - & 3.98 \\
srep-rep2 & 3.98 & 3.99 \\
direct & 1.0 & 1.0 \\
history & 30.28 & 59.61 \\
flood & 68.0 & 132.0 \\
\hline
\end{tabular}

Table 3: Routing overhead of different forwarding algorithms for two node densities. Forwarding algorithms (such as ec and srep) which employ replication only at the source has significantly lower overhead. flood has almost an order of magnitude higher overhead and does not scale well as the number of nodes increase. The high overhead of history results from our implementation in dtnsim2 where a copy of message is transmitted even when some copy of the original message has been delivered. Some timeout scheme can solve this problem by reducing unnecessary message transmissions.

their overhead is constant. For an algorithm with a replication factor of 2, the overhead should be 4, with 2 from the source to the relay and from the relay to the destination and the other 2 for the other relay. On the other hand, in both history and flood where relays also forward to other relays (and there are no restrictions on replication factor), multiple identical copies of the original message are transmitted even after the first delivery of the original message. As Table 3 shows, normally history has a higher overhead than srep and ec. This situation becomes worse when more contacts are available and very likely, more duplicate messages will be transmitted. For flood, almost all the nodes could receive a copy potentially and the overhead is proportional to $2 n$, where $n$ is the number of nodes. The factor of two comes because every relay sends to the destination (even if the destination has already received the message) in our implementation. Some simple timeout scheme, such as one that imposes a maximum number of hops a message can traverse, can alleviate this problem. However, data delivery rate will decrease if the number of hops a message can traverse is too small. The exploration of such a trade-off is part of our future work.

In summary, in terms of routing overhead, ec and srep scale well with node density and network size, while flood does not.

\subsubsection{Data success rate}

\begin{tabular}{|c|ccccc|}
\hline Algorithm & 0.25 day & 1 day & 2 days & 4 days & 8 days \\
\hline ec-rep2-p8 & $22.6 \%$ & $95.9 \%$ & $100 \%$ & $100 \%$ & $100 \%$ \\
ec-rep2-p16 & $9.2 \%$ & $94.6 \%$ & $100 \%$ & $100 \%$ & $100 \%$ \\
srep-rep2 & $51.8 \%$ & $92.5 \%$ & $99.6 \%$ & $99.9 \%$ & $99.9 \%$ \\
direct & $32.0 \%$ & $74.6 \%$ & $94.2 \%$ & $99.5 \%$ & $99.9 \%$ \\
history & $58.4 \%$ & $87.9 \%$ & $92.7 \%$ & $94.6 \%$ & $95.3 \%$ \\
flood & $100 \%$ & $100 \%$ & $100 \%$ & $100 \%$ & $100 \%$ \\
\hline
\end{tabular}

Table 4: Data success rate of different algorithms for different deadlines. Even with extremely large deadline of 8 days simple replication can not transfer all its data. Also note that, ec has low data success rate when deadlines are extremely small and hence, caution must be used before deciding to use erasure coding.

Table 4 shows the data success rate for different algorithms with

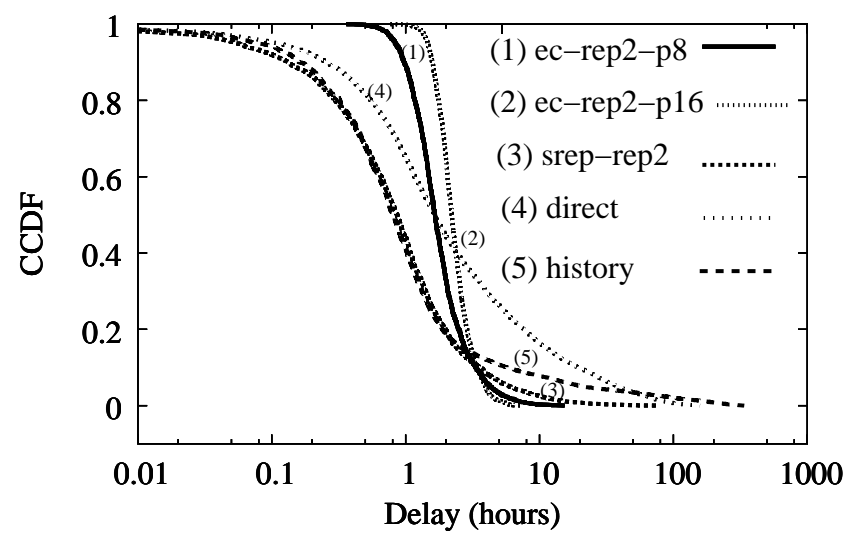

Figure 3: Latency distribution of different forwarding algorithms for the Pareto trace. We use a log-scaled $x$-axis for clarity. Similar to the ZebraNet trace we observe that tails are significantly smaller when ec is used, i.e., the worst case delays for other approaches are significantly higher. Since $x$-axis is log scale, the ratio of the worst case delay values is higher than in the ZebraNet trace.

deadlines smaller than the total simulation time. All deadlines are specified in units of days. The data success rate for ec is low if the deadlines are less than 6 hours long. However, for relatively long deadlines (between 1 and 2 days), ec has the highest data success rate. This result can be observed directly by looking at the data latency distribution curve. Because ec has a lower $99^{t h}$ percentile of latency distribution, it will deliver more messages before that time and hence a higher data success rate. Therefore, if achieving low latencies for all messages or high success rate within certain reasonable deadlines are the application requirement, ec should be used.

On the other hand, history has the highest data success rate when the deadline is less than 6 hours. This is because history can find good relays without the need to distribute copies of data to many relays. The performance improvement of history upon direct and srep comes directly from the efficiency of its selection of good relays. However, since history has long tails in its data latency distribution curve, its data success rate is relatively low compared to other approaches.

\subsection{Impact of mobility model}

In this section, we evaluate the performance of ec and other approaches on a different mobility model. Our results here demonstrate that the idea of using erasure-coding based routing can be applied to different scenarios other than the ZebraNet trace. We find that the benefits of erasure coding are greater when the intercontact times are heavy-tailed. We use such a heavy-tailed distribution for simulations in this section. The mobility model is based on the approximate power law distribution for inter-contact times observed for another set of real-world traces described in [2].

Figure 3 plots the CCDF of the data latency distribution for the Pareto trace. The other simulation parameters are exactly the same as in Section 4.1. Observe that all curves are much sharper than the ZebraNet traces. Again, ec has the sharpest CCDF curve and the lowest $99^{\text {th }}$ percentile delay, while all the other algorithms have higher worst case delays.

\section{DELAY DISTRIBUTION ANALYSIS}

This section discusses the theoretical behavior of the delay dis- 
tribution when the erasure-coding based approach is used. We also theoretically compare the simple replication approach with the erasure-coding based approach.

\subsection{Network model and assumptions}

We consider the following scenario. Consider a source and destination pair which can communicate via one of the $n$ distinct relays. Delay seen if relay $i$ is used is a random variable with distribution $X_{i}$. Using a random variable allows us to model the fact that the delay encountered in using a relay is not known precisely. We assume the delay variables $\left(X_{i}\right)$ to be identical and independent. Although a strong assumption, this should be seen as a first step towards a more sophisticated analysis. Even with these assumptions, we show interesting behavior which gives fundamental insights on the potential of using an erasure-coding based approach. We now introduce the concept of order statistics, the mathematical theory that is used to derive results.

\subsection{Order statistics background}

Consider $n$ identical and independent (IID) random variables $X_{1}, X_{2} \ldots X_{n}$. Let $Y_{1}^{n}, Y_{2}^{n}, \ldots Y_{n}^{n}$ be the random variables obtained by sorting the set $X_{1}, X_{2} \ldots X_{n}$ in increasing order. The random variable $Y_{k}$ is called the $k^{t h}$ order statistic. ${ }^{2}$ One can obtain the distribution of $Y_{k}$ in the following form [12]. Let $F(x)$ be the cumulative distribution function (CDF) of the variables $X_{i}$ and $f(x)$ be the probability distribution function (PDF) $\left(X_{i}\right.$ are identical). Then the PDF (denoted by $\left.g_{k}^{n}(y)\right)$ of $Y_{k}^{n}$ is given by:

$$
g_{k}^{n}(y)=f(y) \frac{n !}{(k-1) !(n-k) !} F(y)^{k-1}(1-F(y))^{n-k}
$$

\subsection{Results for the delay distribution}

In the erasure-coding based approach, the source erasure-codes a message and divides the code blocks equally among $n$ relays. For simplicity, we assume that $n$ is a multiple of $r$, and $n=r k$. A destination can decode a message as soon as $k$ relays successfully deliver data. Let $E C_{r}^{n}$ denote the delay distribution when ec is used with $r$ replication and $n$ relays. Using the terminology of order-statistics $E C_{r}^{n}$ is defined as the $k^{t h}$ order statistic over the variables $\left\{X_{1}, X_{2}, \ldots, X_{n}\right\}$.

$$
E C_{r}^{n}=Y_{k}^{n}
$$

In simple replication, $r$ relays are used and the message can be decoded when one of these relays successfully delivers data. Although the choice of relays could vary with the amount of information available to the forwarding algorithm, for simplicity we assume that the relays $1 \ldots r$ are used by the simple replication approach. Therefore, for simple replication, the observed delay is the minimum of the random variables $\left\{X_{1}, X_{2}, \ldots, X_{r}\right\}$.

$$
\operatorname{srep}_{r}=Y_{1}^{r}
$$

Equation 1 (for distribution of the $k^{\text {th }}$ order statistic) can now be used to obtain the delay distribution. However, it is hard to directly compare the statistics of these distributions from the above formula. In the rest of this section, we discuss two aspects based on the above analysis. First, we discuss the behavior of $E C_{r}^{n}$ as a function of $n$ ( $n$ measures how aggressively erasure coding is used). In particular, we derive the asymptotic distribution for $E C_{r}^{n}$ (when $n \rightarrow \infty$ ). Second, we compare srep and $E C_{r}^{n}$ analytically, for both finite $n$ and as $n \rightarrow \infty$.

$$
\overline{{ }^{2} Y_{1}^{n} \equiv \min _{j}\left(X_{j}\right) \text { and } Y_{n}^{n}} \equiv \max _{j}\left(X_{j}\right) .
$$

\subsection{Understanding the nature of $E C_{r}^{n}$}

The exact distribution of $E C_{r}^{n}$ depends on the distribution of $X_{i}$. For our analysis, we consider the case when $X_{i}$ are given by the Pareto distribution. Recent work on mobility analysis has hinted that such delay distributions are heavy-tailed and thus it is an interesting case to discuss in-depth [2]. We also briefly consider the more traditional case when $X_{i}$ are exponential (for example, when the underlying mobility model is random waypoint).

For a Pareto distribution, the probability distribution function is given by $f(x)=\left(b^{\alpha}\right) \alpha x^{-\alpha-1}$. The constant $b$ is its scale parameter, and $\alpha$ is the power law coefficient. For $\alpha<1, E[X]=\infty$ (i.e., using only one relay will have an infinite average delay). ${ }^{3}$

To understand the nature of $E C_{r}^{n}$, we plot the PDF of $E C_{r}^{n}$ for different values of $n$. Figure 5.4 shows the PDF and CDF respectively. We consider the case when $\alpha=.6$. The choice of $\alpha$ is arbitrary and the only important aspect is that it presents a case when the distribution is extremely heavy-tailed.

Key Observations. As $n$ increases, the distribution becomes thinner and more bell-shaped. In particular, the variance of the distribution reduces as $n$ increases. Low variance means that the probability of encountering very large delays due to bad choice of relays is reduced. Also note that the mean delay reduces as $n$ increases. We find that with $n=32$, the mean delay is within $10 \%$ of the delay obtained when $n=\infty$ relays are used. This shows that convergence is reasonably quick, and most of the advantages of erasure coding can be obtained by using a moderate number of relays.

The following result formalizes the observations made above. The proof is based upon limiting analysis of quantiles of large number of independent variables and is beyond the scope of this paper [12].

RESULT 1. Let the underlying delay variables $X_{i}$ have a continuous PDF $f(x)$. Let $\zeta_{\frac{1}{r}}$ denote the $\frac{1}{r}^{\text {th }}$ quantile of the random variable $X_{i} .{ }^{4}$ Then for sufficiently large $n$, the distribution of $E C_{r}^{n}$ converges to the normal distribution in the following manner:

$$
E C_{r}^{n} \text { is } \mathcal{N}\left(\zeta_{\frac{1}{r}}, \frac{(r-1)}{n f^{2}\left(\zeta_{\frac{1}{r}}\right)}\right)
$$

COROLlary 1.

$$
\begin{aligned}
\lim _{n \rightarrow \infty} E\left[E C_{r}^{n}\right] & =\zeta_{\frac{1}{r}} \\
\lim _{n \rightarrow \infty} \text { Variance }\left[E C_{r}^{n}\right] & =0
\end{aligned}
$$

It follows immediately that, for large $n$, the distribution of $E C_{r}^{n}$ converges to a constant. The mean delay is $\frac{1}{r}^{t h}$ quantile of the original distribution. This result is also interesting because it indicates that only the $\zeta_{\frac{1}{r}}$ of the original distribution is relevant to characterize the performance when erasure coding is used.

Corollary 2. When $X_{i}$ have Pareto distributions with scale parameter $b$ and power-law coefficient $\alpha, E\left[E C_{r}^{n}\right]$ is given by,

$$
\lim _{k \rightarrow \infty} E\left[E C_{r}^{n}\right]=b\left(\frac{r}{r-1}\right)^{\frac{1}{\alpha}}
$$

\footnotetext{
${ }^{3} E[\cdot]$ is used to denote expectation of a random variable.

${ }^{4}$ By definition, $\operatorname{Prob}\left(X_{i}<\zeta_{\frac{1}{r}}\right)=\frac{1}{r}$. $\zeta_{\frac{1}{r}}$ is obtained by the inverse $\mathrm{CDF}$ function.
} 


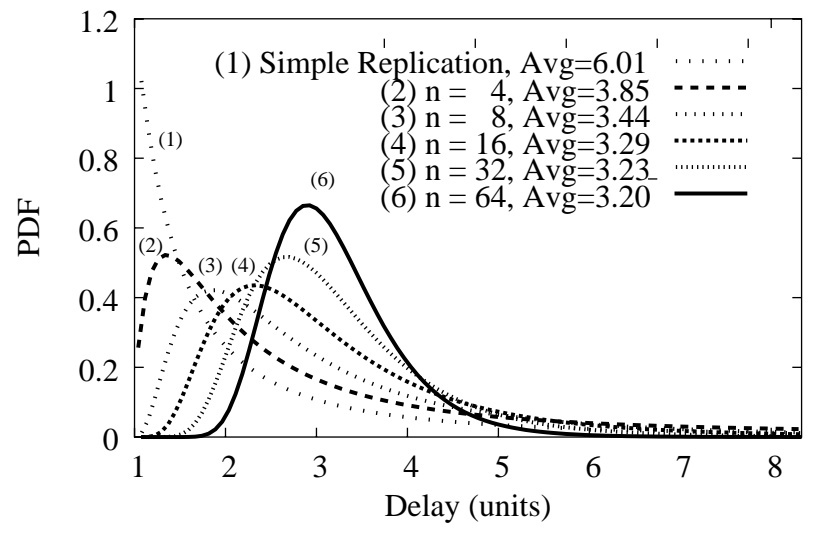

(a) Probability Distribution Function (PDF)

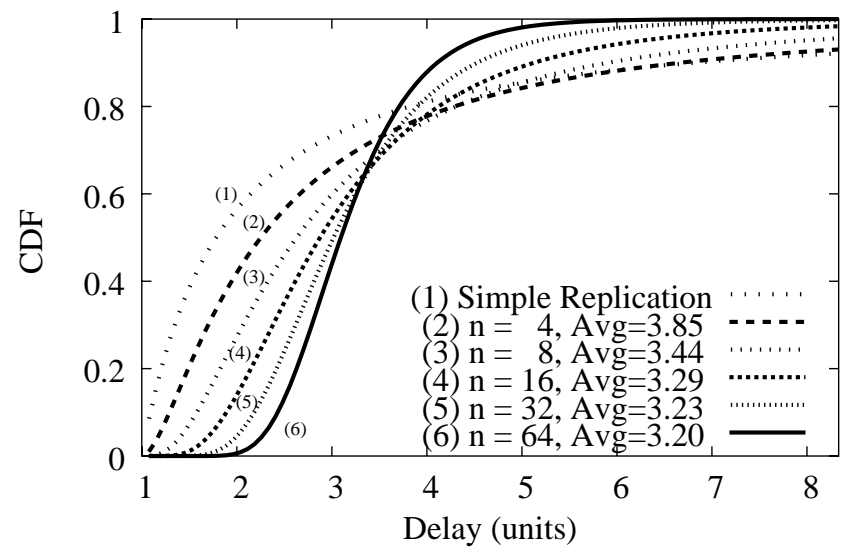

(b) Cumulative Distribution Function (CDF)

Figure 4: PDF and CDF of the delay distribution for different values of $n$ when using the erasure-coding based approach. Underlying delay distribution is Pareto with $\alpha=.6$, and $b=1$. $r=2$. For each line, the value after the label $A v g$ denotes the average value of the distribution $\left(E\left[E C_{r}^{n}\right]\right)$. We observe that as $n$ increases the distribution becomes thinner and more bellshaped.

The above equation shows that for any value of $\alpha$, the delay behavior of an erasure-coding based approach converges to a constant (for any finite amount of replication factor $r$, such that $r>1$ ). This is particularly interesting because for $\alpha<1$, the delay of using any single relay by itself is $\infty$. In fact, the srep algorithm, which uses the first $r$ relays, will also have an infinite delay on average if the product $r \alpha<1$. For this case, the erasure-coding based approach has infinitely better performance. This shows that for heavy-tailed delay distributions, the erasure-coding based approach is able to get rid of the tail (i.e long delays) whereas simple replication can not.

For the Pareto case, we can also derive the value of $E\left[E C_{r}^{n}\right]$ for a finite value of $n$. This is given by the following expression,

$\frac{b}{E\left[E C_{r}^{n}\right]}=\left(1-\frac{1}{n \alpha}\right)\left(1-\frac{1}{(n-1) \alpha}\right) \ldots\left(1-\frac{1}{(n-(n / r)+1) \alpha}\right)$

This expression is useful in determining the value of $n$ at which the expected delay converges to its eventual value $\zeta_{\frac{1}{r}}$.

\subsection{Comparing ec and srep}

We argued above that the delay distribution when using erasure

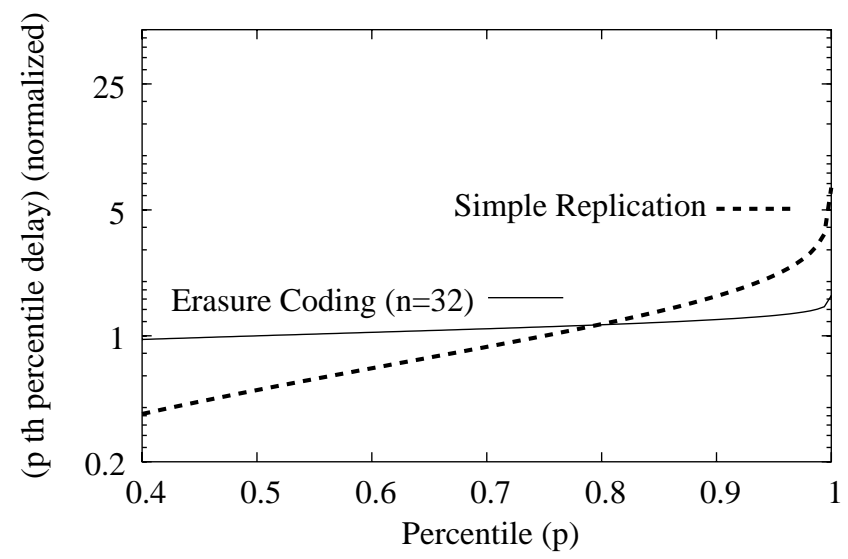

(a) $X_{i}$ are Exponential

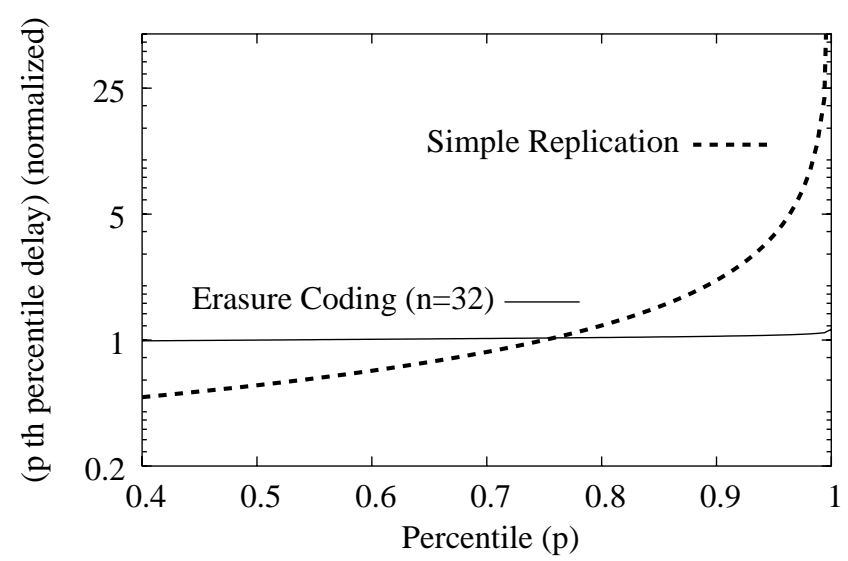

(b) $X_{i}$ are Pareto

Figure 5: Comparing percentiles delay for ec and srep. $r=2, n=32$. a) $X_{i}$ are exponential b) $X_{i}$ are Pareto $(\alpha=.6)$. Delays are normalized to the $\zeta_{\underline{1}}$ of the underlying distribution $\left(X_{i}\right)$, which makes the plots independent of the scale parameters of the underlying distribution. The plot is obtained by first determining delay distributions in accordance to Equation 2 for the ec approach and Equation 3 for the simple replication approach. The percentiles are then analytically computed by inverting the cumulative distribution function. ec approach performs significantly better than srep when comparing higher percentiles of the resultant delay distribution. In fact, ec approach has almost non increasing delay values for different percentiles.

coding has low variance and therefore, it has lower worst case delay than simple replication. To support this, we compare the $p^{t h}$ percentile delay for ec and srep. The $p^{t h}$ percentile delay for a random delay variable $Z$ is defined as the value $w$ such that $\operatorname{Prob}(Z \leq w)=p$. Figure 5 compares percentiles delay for srep and ec for two underlying delay distributions $\left(X_{i}\right)$, a) Exponential, b) Pareto with $\alpha=.6 . r=2$ for both srep and ec, and $n=32$ for ec. The delay values for each approach are normalized to the $\zeta_{\frac{1}{r}}$ of the underlying delay distribution $X_{i}$. This makes the plots independent of the scale parameters of the underlying distribution.

For lower percentiles, the erasure-coding based approach has higher delays. This implies that for the few best cases, simple repli- 
cation has lower delays than ec. However, the delays observed with ec towards the higher percentiles are much lower than srep. For ec, even for a moderate value of $n=32$, delay values across percentiles are more or less the same, and close to the $\zeta_{\underline{1}}$. For simple replication, delays for higher percentiles are very high. In this example, for the Pareto case, the $99^{t h}$ percentile delay for srep is 25 times more than the erasure-coding based approach.

\section{RELATED WORK}

A series of efforts $[8,14,9]$ in the context of sensor and mobile ad-hoc networks explore various forwarding algorithms for opportunistic networks. These techniques employ a form of data duplication (typically based on controlled flooding) to achieve eventual delivery.

The ZebraNet system uses a history-based protocol that leverages the contact history of nodes to the base station [8]. Simple replication approach which uses only one relay was shown to achieve optimal throughout in a mobile ad-hoc network [3] and has been further analyzed in [7, 2].

Erasure codes have recently been discussed and applied to many network applications, including achieving efficient distribution of bulk data in overlay networks [1] and P2P networks [10], coping with unreliable transmissions in wireless sensor networks [13], and achieving reliability in large-scale distributed storage systems [4]. Applying erasure coding to combat uncertainty in delay performance is the focus of our work. Since the idea of erasure-coding based forwarding is orthogonal to all other forwarding approaches, it can potentially be combined with them.

\section{CONCLUSIONS AND FUTURE WORK}

We presented a novel forwarding algorithm based on the use of erasure coding for opportunistic networks. The use of erasure coding spreads the responsibility of forwarding over many nodes while maintaining a fixed overhead. We showed using both analysis and simulation against a real-world mobility trace that our erasurecoding based approach significantly improves the worst case delay. At the same time, it has no "very small delay" cases. This is a natural consequence of how this approach works. We believe that the basic idea holds promise and an approach that combines erasure coding with other techniques, such as simple replication, may give us good performance on both fronts.

We have introduced the erasure coding idea in a simple setting in which all nodes are equally good relays. If different nodes have different characteristics, a more sophisticated approach to spread erasure code blocks is required. This is part of our ongoing effort and is discussed in [5]. We also plan to investigate the utility of the erasure coding approach on more mobility traces with diverse characteristics. Our analysis is preliminary and assumes that different relays used for forwarding data have independent delay distributions. This assumption breaks down if erasure coding uses the best $n$ relays. Extending our analysis to incorporate such cases is an interesting future direction. Finally, based on the observation that different approaches have distinct advantages under certain mobility characteristics, it is desirable to have an adaptive strategy that selects one routing scheme over another on-the-fly, trying to achieve the best aspects of various forwarding algorithms.

\section{ACKNOWLEDGMENTS}

We gratefully acknowledge Andre Adler from IIT Chicago for pointing us to Sterfling [12] for the results regarding the distribution of order-statistics.

\section{REFERENCES}

[1] J. Byers, J. Considine, M. Mitzenmacher, and S. Rost. Informed Content Delivery Across Adaptive Overlay Networks. In Procs. of ACM SIGCOMM, 2002.

[2] A. Chaintreau, P. Hui, J. Crowcroft, C. Diot, R. Gass, and J. Scott. Pocket Switched Networks: Real-World Mobility and its Consequences for Opportunistic Forwarding. Technical Report UCAM-CL-TR-617, University of Cambridge, Computer Laboratory, February 2005.

[3] M. Grossglauser and D. Tse. Mobility Increases the Capacity of Ad-hoc Wireless Networks. Transactions on Networking, vol. 10, no. 4, Aug. 2002.

[4] Hakim Weatherspoon and John Kubiatowicz. Erasure Coding vs. Replication: A Quantitative Comparison. In Procs. of IPTPS, 2002.

[5] S. Jain, M. Demmer, R. Patra, and K. Fall. Using Redundancy to Cope with Failures in a Delay Tolerant Network. In Procs. of ACM SIGCOMM, 2005.

[6] S. Jain, K. Fall, and R. Patra. Routing in a Delay Tolerant Network. In Procs. of ACM SIGCOMM, 2004.

[7] S. Jain, R. Shah, W. Brunette, G. Borriello, and S. Roy. Exploiting Mobility for Energy Efficient Data Collection in Sensor Networks. In Procs. of WiOpt, 2004.

[8] P. Juang, H. Oki, Y. Wang, M. Martonosi, L.-S. Peh, and D. Rubenstein. Energy-Efficient Computing for Wildlife Tracking: Design Tradeoffs and Early Experiences with ZebraNet. In Procs. of ACM ASPLOS, 2002.

[9] A. Lindgren, A. Doria, and O. Schelén. Probabilistic Routing in Intermittently Connected Networks. In Procs. of SAPIR, 2004.

[10] P. Maymounkov and D. Mazieres. Rateless Codes and Big Downloads. In Procs. of IPTPS, 2003.

[11] M. Mitzenmacher. Digital Fountains: A Survey and Look Forward. Information Theory Workshop, 2004.

[12] R. J. Sterfling. Approximation Theorems of Mathematical Statistics. Wiley Series in Probability and Statistics, 1980.

[13] Sukun Kim and Rodrigo Fonseca and David Culler. Reliable Transfer on Wireless Sensor Networks. In Procs. of IEEE SECON, 2004.

[14] A. Vahdat and D. Becker. Epidemic Routing for Partially Connected Ad Hoc Networks. Technical Report CS-200006, Duke University, 2000.

[15] P. Zhang, C. M. Sadler, S. Lyon, and M. Martonosi. Hardware Design Experiences in ZebraNet. In Procs. of ACM Sensys, 2004. 